\title{
Patient education and informed consent for preimplantation genetic diagnosis: Health literacy for genetics and assisted reproductive technology
}

\author{
Michelle L. McGowan, PhD ${ }^{1}$, Christopher J. Burant, PhD', Rocio Moran, $M D^{3}$, and \\ Ruth Farrell, $M A, M D^{4}$
}

\begin{abstract}
Purpose: Innovative applications of genetic testing have emerged within the field of assisted reproductive technology through preimplantation genetic diagnosis. As in all forms of genetic testing, adequate genetic counseling and informed consent are critical. Despite the growing recognition of the role of informed consent in genetic testing, there is little data available about how this process occurs in the setting of preimplantation genetic diagnosis. Methods: A cross-sectional study of in vitro fertilization clinics offering preimplantation genetic diagnosis in the United States was conducted to assess patient education and informed consent practices. Descriptive data were collected with a selfadministered survey instrument. Results: More than half of the clinics offering preimplantation genetic diagnosis required genetic counseling before preimplantation genetic diagnosis $(56 \%)$. Genetic counseling was typically performed by certified genetic counselors (84\%). Less than half $(37 \%)$ of the clinics required a separate informed consent process for genetic testing of embryonic cells. At a majority of those clinics requiring a separate informed consent for genetic testing (54\%), informed consent for preimplantation genetic diagnosis and genetic testing took place as a single event before beginning in vitro fertilization procedures. Conclusions: The results suggest that patient education and informed consent practices for preimplantation genetic diagnosis have yet to be standardized. These findings warrant the establishment of professional guidelines for patient education and informed consent specific to embryonic genetic testing. Genet Med 2009:11(9):640-645.
\end{abstract}

Key Words: genetic testing, preimplantation genetic diagnosis, informed consent

$\mathrm{T}$ he latter half of the 20th century witnessed the rapid advancement of genetic testing. A variety of genetic tests have become readily available and accessible including prenatal testing and newborn screening in addition to diagnostic and carrier testing for children and adults. More recently, genetic testing has emerged within the field of assisted reproductive technology (ART) through the application of genetic testing to the tech-

From the ${ }^{1}$ Department of Bioethics, Center for Genetic Research Ethics and Law, Case Western Reserve University School of Medicine; ${ }^{2}$ Frances Payne Bolton School of Nursing and Department of Bioethics, Case Western Reserve University; ${ }^{3}$ Center for Personalized Genetic Healthcare, Cleveland Clinic Foundation; and ${ }^{4}$ Department of Surgery, Case Western Reserve University Lerner College of Medicine and Departments of Bioethics and Obstetrics/Gynecology, Cleveland Clinic Foundation, Cleveland, Ohio.

Ruth M. Farrell, MA, MD, Department of Bioethics, Cleveland Clinic, 9500 Euclid Avenue, JJ-60, Cleveland, OH 44195. E-mail: farrelr@ccf.org.

Disclosure: The authors declare no conflict of interest.

Submitted for publication December 22, 2008

Accepted for publication April 9, 2009.

Published online ahead of print July 31, 2009.

DOI: $10.1097 /$ GIM.0b013e3181ac6b52 nique of preimplantation embryo biopsy, a procedure referred to as preimplantation genetic diagnosis (PGD).

PGD combines the techniques of in vitro fertilization (IVF) and embryo micromanipulation with genetic testing to determine the hereditary characteristics of an embryo. The advantage of PGD is that this genetic information is made available before the embryo is transferred to a women's uterus and becomes an established pregnancy. ${ }^{1}$ The procedure of PGD entails two composite steps. First, embryo biopsy is performed to obtain one or two embryonic cells (blastomeres) for analysis. Second, genetic tests are performed on these embryonic cells. Embryos identified with a characteristic of interest may then be used or withheld for uterine transfer. This procedure presents prospective parents with an alternative to invasive prenatal testing while avoiding the risks of possible fetal injury and iatrogenic miscarriage in addition to having to make decisions about continuing or terminating an affected pregnancy. PGD has also been introduced as a way to increase pregnancy rates from ART by screening for chromosomal aneuploidy or familial disorders before embryo transfer. ${ }^{2,3}$ The utilization for PGD is advancing rapidly. Recent estimates suggest that PGD is available at $74 \%$ of fertility clinics surveyed in this country, although this genetic test is only used in about $4 \%$ to $6 \%$ of IVF cases at those clinics, resulting in about 3000 IVF-PGD procedures conducted in the United States in 2005. ${ }^{4}$

Typically, reproductive genetic testing arises in the context of preconception testing or prenatal testing of an existing pregnancy. PGD falls in between these two types of testing on the reproductive timeline, as conception has already occurred yet the pregnancy has not yet been established within a woman's uterus. Nevertheless, the importance of achieving informed consent for this type of reproductive genetic testing remains the same. Recognizing that elements of both preconception and prenatal genetic testing exist in the context of PGD illustrates the need to situate PGD on the continuum of genetic testing for reproductive decision making. Yet, it is also important to distinguish PGD from other forms of genetic technology because there are unique and challenging factors to consider when performing PGD. The informed consent process for PGD presents several challenges, many of which pertain to the complicated medical technology involved in embryo creation and genetic testing on embryonic cells, techniques that are not a part of other forms of reproductive genetic testing.

PGD is a hybrid procedure, which brings together the technological advances of ART and genomic medicine. On the one hand, PGD is an ART procedure, entailing the retrieval of human oocytes, in vitro generation of embryos, and removal of individual cells for testing. Each of these procedures has its own set of risks and benefits both for the woman and the future child. ${ }^{1,5}$ On the other hand, PGD is a genetic testing procedure. Just as with other forms of reproductive genetic testing, it is imperative that prospective parents understand the risks, benefits, and implications of learning about a genetic characteristic 
for both their own health and that of their future child. This information is particularly critical in the current context of genetic technology, specifically because much uncertainty remains about the significance, prognosis, and management of a genetic mutation once it is identified. ${ }^{6}$ Although PGD shares commonalities with other forms of genetic testing modalities, it also has a set of important and distinct differences. Unlike other forms of genetic testing in which multiple cells are available for analysis, embryo biopsy produces only one or two cells. As a result, the likelihood of misdiagnosis or uncertain diagnosis may be greater than in standard testing procedures. Minute and limited samples of DNA can be acquired on a one-time basis for analysis, and nondiagnostic results cannot be resolved with repeat embryo biopsy. ${ }^{7}$

Such advances in ART raise several questions about how to integrate genetic testing into reproductive medicine. An important set of these questions pertains to patients' ability to make informed decisions about the use of genetic tests in the context of reproduction and ART. Health literacy, a growing field of study, plays a vital role in how patients use complex health care information to make medical decisions. Informed consent, one aspect of health literacy, is a process of communication by which patients obtain pertinent medical information about the indications, risks, benefits, and alternatives of the proposed treatment from their health care provider. Using this education, patients then can make informed and voluntary choices to accept or decline the procedure. Informed consent is a cornerstone of clinical medicine. As an ethical construct, informed consent is a mechanism whereby patients are able to make autonomous choices about their health care. As a performance construct, informed consent is directly related to the quality of health care and to the patient safety.

It has been well recognized that informed consent plays a crucial role in genetic testing. Professional guidelines, such as those of the National Society of Genetic Counselors, emphasize the importance of achieving adequate informed consent before proceeding with genetic testing, and that the process should be conducted by a board-certified genetic counselor or an individual with equivalent knowledge and experience. ${ }^{8}$ The National Society of Genetic Counselors' guidelines for preconception and prenatal genetic screening also emphasize the importance of posttest counseling to ensure that patients have adequate education about the testing process (specifically information about the test's specificity, sensitivity, accuracy, risks, benefits, and limitations), any follow-up diagnostic tests, as well as patients reproductive options in the event of a positive diagnostic test result. ${ }^{9}$

As a hybrid procedure, an informed consent process should include a discussion of both aspects of the PGD process: (1) the procedures involved to obtain embryonic cells and (2) the genetic testing process. However, there are few guidelines or recommendations available to assist health care providers or patients in these processes. Professional organizations, such as the European Society of Human Reproduction and Embryology PGD Consortium, have established a set of guidelines for achieving informed consent for PGD, which is currently the most robust model available. ${ }^{10}$ Motivated by the need for practice standardization and improved quality of health care, these guidelines recommend that separate informed consent should be obtained for each of the procedures involved in PGD, including those for creating IVF embryo(s), embryo biopsy, and genetic testing of the biopsied cells. Despite these recommendations, there is a paucity of data about the process and quality of the informed consent processes currently taking place in clinical practice.
No standardized data collection mechanisms exist to track informed consent practices among PGD providers in the United States. Furthermore, review of the literature reveals that the processes of informed consent for PGD in clinics in the United States have not been directly analyzed. Although a recent study on the quality assurance practices of 53 PGD centers in 17 European countries reported that $94 \%$ of clinics required informed consent for PGD, there was discrepancy reported regarding how to conduct the process of patient education and informed consent in addition to uncertainty as to whether or not patients were adequately counseled for PGD. ${ }^{11}$ A retrospective study of patient experiences with PGD procedures conducted in the United States raised similar concerns that deficiencies in patient understanding exist, particularly pertaining to the indication, risks, and limitations of the technical aspects of PGD. ${ }^{12}$

From the few studies assessing patients' experiences with PGD, it is evident that some patients have had reservations about the quality of educational content and the informed consent process before proceeding with PGD. ${ }^{13-15}$ One qualitative study of PGD patients in the United Kingdom indicated that they felt well informed regarding the technical and emotional difficulties of IVF and PGD. ${ }^{16}$ However, another study conducted in the United States revealed that PGD patients felt underinformed about several key aspects of the procedure, namely the procedure's low take-home-baby rate, limitations, accuracy, and reliability of the genetic tests developed and used for PGD, and patients' obligation to undergo subsequent invasive genetic testing during the resulting pregnancy. ${ }^{13}$

Given the importance of informed consent in the practice of modern medicine and insight into patients' experiences with this new technology, it is important to examine how patient decision making is taking place for PGD. The need for information about how informed consent is obtained is pressing, especially given the quickly growing scope of clinical applications of this technique. In a move toward measuring foundational data about health literacy in the context of reproductive genetic testing, this study is the first to directly analyze the content of patient education and the process of informed consent for PGD at clinics in the United States. The goal of this work is to provide a baseline needs assessment and direction for standardizing adequate processes of facilitating patient decision making for new applications of reproductive genetic testing.

\section{MATERIALS AND METHODS}

This was a pilot study designed to collect descriptive data about the educational content and process of informed consent for PGD, with specific emphasis on the genetic testing aspects of the procedure.

\section{Recruitment}

Before initiation of the study, the protocol received approval from the Cleveland Clinic Institutional Review Board. Because of the nature of this study, written informed consent for participation was not required. Fertility clinics in the United States were identified by means of the Centers for Disease Control and Prevention's 2005 ART Report. ${ }^{17}$ In total, 381 ART clinics were contacted by phone to determine whether PGD services were offered or performed. Of those contacted, 85 IVF clinics responded to this inquiry and stated that they offered PGD services to patients. Information about PGD services could not be obtained from the remainder of the clinics identified by the ART Report either because the staff could not be contacted via phone or the contacted personnel declined to provide the requested information. 
All clinics that reported the current availability of PGD services were contacted to inquire about participation in the study. An introductory e-mail discussing project, objectives, and research personnel was sent to each clinic in advance of survey instrument distribution. No incentives were offered for participation. Within 1 week, all clinics as identified earlier were sent a survey instrument and a copy of the introductory letter with a stamped, addressed envelope to return the completed instrument to the investigators. This packet was addressed and mailed directly to the medical director of each clinic. Thirty days after the initial mailing, the investigators attempted to recontact nonresponders by means of a reminder e-mail and mailing a second paper copy of the original survey instrument. No additional attempts were made to reach nonresponders. Data collection were conducted from August to December 2007.

\section{Instrumentation}

A descriptive survey instrument was developed to assess the content of patient education and the process of informed consent offered to patients before undergoing PGD, with emphasis on the informed consent process for the genetic testing aspect of the procedure. This questionnaire was not designed to assess the specific materials or content of typical information consent discussions, rather it was meant to catalog the components, steps, and parties involved in the informed consent process.

This was a self-administered instrument requiring approximately 10 minutes to complete. It contained several close-ended questions followed by multiple options from which the respondent could choose the response that best described demographic characteristics and clinical practices. Respondents also had the option of providing additional responses if the provided choices insufficiently described their individual practices. Items were written to assess clinic demographics, the role of genetic counselors in PGD services, and the components and process of achieving informed consent for IVF and PGD. When addressing issues specific to informed consent for genetic testing, items were developed to assess the use of genetic counseling services before and after PGD and to catalog the personnel who provided this counseling services. Items were also designed to examine the component parts of informed consent, namely the process of patient education about indications, risks, benefits, and alternatives of PGD. As part of the pilot testing process, a series of additional questions were included in the survey to assess instrument usability.

\section{Data analysis}

Quantitative data from the instruments were coded and entered into a database. All statistics were run using SPSS 15.0. Because the study was an exploratory pilot study, the current analyses focused on describing facility characteristics, genetic counseling services, and informed consent process used in these clinics. For this reason, no power calculations were performed to determine sample size. Frequencies were used for all analyses. The number of participants and percentages for the responses to each question are provided.

\section{RESULTS}

Of the 85 clinics that were successfully contacted, 39 clinics completed and returned the survey instrument giving a response rate of $45.9 \%$. No data were available about demographics or clinical practices of nonresponding clinics. Table 1 provides information on the clinics' characteristics. Twenty-eight (72\%) of the clinics were private practice based and $10(26 \%)$ were
Table 1 Facility characteristics

$N(\%)$

Facility characteristics

Academic/university based

Private practice

Other

No. patients provided with PGD in the last year

$51-100$

$101-200$

$>200$

No. years PGD has been performed at institution (yr)

$0-1$

$1-5$

$5-10$

$>10$

In-house performance of genetic testing

All of the time

Most of the time

Some of the time

Never (blastomeres are sent out for testing)

academic or university based. During the last year, $26(67 \%)$ of these clinics provided PGD to between 1 and 50 patients, whereas five $(13 \%)$ treated 51 to 100 patients, six $(15 \%)$ treated 101 to 200 patients, and two (5\%) treated more than 200 patients. In terms of clinics' experience with PGD, 20 (51\%) have provided these services for 1 to 5 years, $13(33 \%)$ provided these services for 5 to 10 years, $5(13 \%)$ provided these services for over 10 years, and only 1 began providing this service within the last year. With regard to performing in-house genetic testing (i.e., genetic testing and analysis performed by an on-site laboratory), 29 (74\%) of clinics had never performed this service.

Examination of the educational aspects of informed consent indicated that, overall, the content of patient education appeared to be comprehensive. The risks and limitations of genetic testing in general were addressed by all 39 (100\%) clinics, 38 (97\%) discussed risks and limitations of genetic testing specific to PGD and indications for PGD, 37 (95\%) attended to risks and limitations of PGD, and 34 (87\%) discussed family history.

In taking a closer look at genetic counseling services associated with PGD, this study identified the following findings (Table 2). Genetic counseling was required for all patients at 22 $(56 \%)$ of the clinics. Six $(15 \%)$ of the clinics required counseling most of the time and $10(26 \%)$ required counseling some of the time. Genetic counseling was provided by certified genetic counselors at $32(84 \%)$ clinics, by physicians at $10(26 \%)$ clinics, and by a nurse at $1(3 \%)$ clinic. These categories were not mutually exclusive, with staff members sharing responsibility for patient education and obtaining informed consent. Among those clinics, 14 (36\%) had a PGD genetic counselor on staff. 
Table 2 Genetic counseling services

\begin{tabular}{lr}
\hline & $N(\%)$ \\
\hline Requirement of genetic counseling before PGD & $22(56.4)$ \\
All of the time & $6(15.4)$ \\
Most of the time & $10(25.6)$ \\
Some of the time & $1(2.6)$ \\
Never & \\
Provider of genetic counseling services & \\
Certified genetic counselor & $32(84.2)$ \\
Yes & $6(15.8)$ \\
No & $10(26.3)$ \\
Physician & $28(73.7)$ \\
Yes & \\
No & $14(35.9)$ \\
Nurse & $25(64.1)$ \\
Yes & $37(97.4)$ \\
No & \\
Other & \\
Yes & \\
No & \\
No & \\
\hline
\end{tabular}

The education process for genetic testing of embryos was primarily provided through discussion with a member of the clinic team (Table 3). Education about genetic testing of blastomeres involved discussion with a patient's primary physician at eight $(22 \%)$ clinics, a genetic counselor at six (17\%) clinics, a nurse at five (14\%) clinics, an another physician on staff at two $(6 \%)$ clinics, an IVF educator at two $(6 \%)$ clinics, or an IVF embryologist at two (6\%) clinics. In addition, five (14\%) clinics provided pamphlets and other material on genetic testing of blastomeres.

In terms of personnel facilitating the informed consent process, an IVF nurse was the most likely staff member to obtain consent at six (17\%) clinics followed by the patient's physician at five $(14 \%)$ clinics, another physician on staff at one $(3 \%)$ clinic, or an IVF-specific educator at one $(3 \%)$ clinic. These categories were not mutually exclusive. Additionally, 25 (69\%) of the clinics indicated that informed consent must be obtained from both members of the couple presenting for PGD and 11 (31\%) required consent for PGD only from the female patient undergoing IVF.

Examination of the informed consent process indicated that $14(37 \%)$ of the clinics required separate informed consent process for genetic testing of blastomeres (Table 4). In six $(54 \%)$ of those 14 clinics, a single session was conducted to obtain informed consent for all PGD-related procedures at the onset of treatment. In four $(36 \%)$ of these clinics, informed consent for genetic testing was delayed until the time of the
Table 3 Education process for genetic testing of blastomere(s)

\begin{tabular}{|c|c|}
\hline & $N(\%)$ \\
\hline \multicolumn{2}{|l|}{$\begin{array}{l}\text { Methods of education regarding genetic testing of } \\
\text { blastomere(s) }\end{array}$} \\
\hline $\begin{array}{l}\text { Discussion with the patient's/couple's primary } \\
\text { physician }\end{array}$ & $8(22.2)$ \\
\hline Discussion with another physician on staff & $2(5.6)$ \\
\hline Discussion with a nurse & $5(13.9)$ \\
\hline Discussion with an IVF educator & $2(5.6)$ \\
\hline Discussion with a genetic counselor & $6(16.7)$ \\
\hline Discussion with an IVF embryologist & $2(5.6)$ \\
\hline Multimedia presentation & $0(0.0)$ \\
\hline Pamphlets and other written material & $5(13.9)$ \\
\hline Group discussion with other patients & $0(0.0)$ \\
\hline Other & $2(5.6)$ \\
\hline \multicolumn{2}{|l|}{$\begin{array}{l}\text { Information addressed during a genetic } \\
\text { counseling session for PGD }\end{array}$} \\
\hline Indications for PGD & $38(97.4)$ \\
\hline Family history & $34(87.2)$ \\
\hline Risks and limitations of PGD & 37 (94.9) \\
\hline $\begin{array}{l}\text { Risks and limitations of genetic testing in } \\
\text { general }\end{array}$ & $39(100.0)$ \\
\hline $\begin{array}{l}\text { Risks and limitations of genetic testing specific } \\
\text { to PGD }\end{array}$ & $38(97.4)$ \\
\hline Other & $5(12.8)$ \\
\hline
\end{tabular}

embryo biopsy. With regard to assessing if patients understood the disclosed information concerning genetic testing of blastomere, five $(46 \%)$ of these clinics offered no assessment, four (36\%) assessed informally through discussion, and two (18\%) assessed formally with a questionnaire.

\section{DISCUSSION}

This study reports on the content and the processes involved in achieving informed consent for PGD at clinics in the United States. It was designed to assess the status of informed consent procedures for PGD with the intention of providing guidance for improving informed decision making for this rapidly developing clinical application of genetic technology. Although this study has provided informative data about decision making for PGD, there are important limitations to acknowledge. As a pilot project, the study is limited by its small sample size. Nevertheless, the results of the survey have provided descriptive information about how 39 clinics in this country are preparing patients for PGD in addition to laying the groundwork for the design of additional research studies in this area. Although the results of this study are specific to the informed consent practices of a small sample of ART clinics in the United States, the conclusions speak to larger issues that have been raised in the literature regarding the adequacy of informed consent proce- 
Table 4 Informed consent process for genetic testing of blastomere(s)

\begin{tabular}{|c|c|}
\hline & $N(\%)$ \\
\hline \multicolumn{2}{|l|}{$\begin{array}{l}\text { Requirement of a separate informed consent } \\
\text { process for genetic testing of blastomere(s) }\end{array}$} \\
\hline Yes & $14(36.8)$ \\
\hline No & $24(63.2)$ \\
\hline \multicolumn{2}{|l|}{$\begin{array}{l}\text { Timing of informed consent process for genetic } \\
\text { testing of blastomere(s) }\end{array}$} \\
\hline $\begin{array}{l}\text { Single informed consent session for all PGD } \\
\text { related procedures at onset of treatment }\end{array}$ & $6(54.5)$ \\
\hline $\begin{array}{l}\text { At the time the consent is obtained for embryo } \\
\text { biopsy }\end{array}$ & $4(36.4)$ \\
\hline After oocyte retrieval & $0(0.0)$ \\
\hline When the embryo is at the $6-8$ cell stage & $0(0.0)$ \\
\hline After embryo biopsy is performed & $0(0.0)$ \\
\hline Other & $1(9.1)$ \\
\hline \multicolumn{2}{|l|}{$\begin{array}{l}\text { Staff responsible for obtaining informed consent } \\
\text { for genetic testing of blastomere(s) }\end{array}$} \\
\hline Patient's/couple's primary physician & $5(13.9)$ \\
\hline Another physician on staff & $1(2.8)$ \\
\hline Nurse & $6(16.7)$ \\
\hline IVF educator & $1(2.8)$ \\
\hline Other & $3(8.3)$ \\
\hline \multicolumn{2}{|l|}{$\begin{array}{l}\text { Individual(s) involved in the informed consent } \\
\text { process for genetic testing of blastomere(s) }\end{array}$} \\
\hline $\begin{array}{l}\text { Both members of the couple presenting for } \\
\text { PGD }\end{array}$ & $25(69.4)$ \\
\hline $\begin{array}{l}\text { Only the patient who will undergone IVF/PGD } \\
\text { procedures }\end{array}$ & $11(30.6)$ \\
\hline $\begin{array}{l}\text { Only the member of the couple who carries the } \\
\text { genetic mutation of interest }\end{array}$ & $0(0.0)$ \\
\hline \multicolumn{2}{|l|}{$\begin{array}{l}\text { Assessment of patient understanding of disclosed } \\
\text { information about genetic testing of } \\
\text { blastomere(s) }\end{array}$} \\
\hline No assessment & $5(45.5)$ \\
\hline Informally assessed through discussions & $4(36.4)$ \\
\hline $\begin{array}{l}\text { Formally assessed with the use of a } \\
\text { questionnaire }\end{array}$ & $2(18.2)$ \\
\hline
\end{tabular}

dures both for PGD and, more broadly, for reproductive genetic testing. ${ }^{11-15}$

In regard to the content of patient education and informed consent, the surveyed clinics appeared to be consistent in the type of information that they provide to patients. These clinics reported to adhere very closely to professional standards for genetic counseling, including assessing patients' motivations and family pedigree, informing patients about the risks and limitations of genetic testing, providing information about the medical risks and complications associated with PGD, and providing information about the risks and limitations of per- forming genetic testing on a single cell biopsied from an IVF embryo. In addition, a majority of clinics required that both members of a couple be a part of the consent process for PGD. The uniformity of content conveyed in genetic counseling and standard practice of dual consent across the surveyed clinics is commendable and should be continued in informed consent practice.

Despite the findings pertaining to content, there is little consistency across surveyed clinics in terms of the process of patient education and informed consent. The survey results presented here indicate that ART clinics in the United States offering PGD have not standardized how informed consent is achieved. Who is responsible for educating patients about PGD, who confirms that the information is understood, and whether a board-certified genetic counselor or equivalent professional is involved in the process varies from clinic to clinic.

One of the most striking findings was that clinics have varied standards for whether or not they require genetic counseling for patients considering PGD, with only 22 (56.4\%) of clinics reporting that they require genetic counseling all of the time. Furthermore, only $14(35.9 \%)$ of the clinics have a genetic counselor on staff, although clinics indicated that genetic counseling services are offered to patients by a certified genetic counselor at $32(82.1 \%)$ clinics and/or a physician at $10(25.6 \%)$ clinics. In accordance with professional guidelines for other forms of reproductive genetic testing, a board-certified genetic counselor, geneticist, or professional with equivalent experience is the most appropriate individual to educate and to obtain informed consent from patients considering PGD. If professional standards for achieving informed consent for genetic testing are to be met, then the recommendation of counseling as an essential component for patients' decision making about PGD should be a requirement across all clinics that offer this service.

An additional area where improvement in informed consent practices is still needed is in the processes to ensure that patients make informed decisions. At the 14 clinics that required a separate informed consent process for genetic testing of blastomeres, patient understanding, one of the foundational components of informed consent, was either assumed to be adequate at five $(46 \%)$ clinics or only informally assessed at four $(61.5 \%)$ clinics. Furthermore, the data suggest that patients were often confronted with decision making for a series of complex medical procedures all together, introducing an opportunity for misunderstanding. Six (54\%) of the clinics requiring a separate informed consent for genetic testing reported that informed consent for PGD was bundled with those for the prerequisite IVF procedures and, therefore, obtained during a single consent session. In contrast, four $(30.8 \%)$ of these clinics sought informed consent for PGD at a separate time. These data raise the question about the quality of understanding that patients may have before proceeding with PGD. These data may speak to the findings of studies demonstrating that some patients have had reservations about the quality of informed consent provided to them before proceeding with PGD, particularly those who felt underinformed about the limitations, accuracy, and reliability of the genetic testing aspects of the procedure. ${ }^{13-15}$

The rapidly growing use of PGD in reproductive medicine may explain the lack of standardization of informed consent procedures from clinic to clinic. However, the need for standard guidelines for adequately educating patients about PGD and achieving informed consent remains and will become more urgent as the clinical application of PGD continues to grow in ART practice. Given the range of complications to achieving informed consent for PGD, guidelines from professional orga- 
nizations should be available to assist ART providers in helping their patients make informed decisions about this technology. Using the European Society of Human Reproduction and Embryology PGD Consortium guidelines as a starting point, ${ }^{10}$ it would be ideal if patients would provide informed consent for each of the separate procedures that need to be performed in order for PGD to be complete. Building on this guideline, it is our recommendation that the informed consent process for PGD should be provided before the initiation of IVF, so that patients are not thrust into a potentially time-sensitive decision-making situation after IVF has commenced. However, the informed consent process for PGD should not be bundled with those of the prerequisite IVF procedures. Patients should be given the opportunity to consider the indications, risks, benefits, and alternatives of each of these procedures separately so that they may make the most personally suitable decision possible. Furthermore, all individuals providing education about the genetic testing aspect of the PGD procedure should have formal training and experience in clinical genetics. The same individual who educates patients about the indications, risks, and limitations of genetic testing of the blastomere should also conduct the informed consent process so to ensure continuity in patient comprehension and preparedness for moving forward with the testing procedures.

The guidelines for reproductive genetic testing laid out by the profession of genetic counseling should also serve as a template for ART clinics offering PGD. ${ }^{8,9}$ Our hope is that both the assisted reproduction and genetic counseling professions consider the importance of multidisciplinary collaboration to establish standard professional guidelines for achieving informed consent for patients considering PGD. In doing so, such guidelines would help ensure that all patients are adequately advised of the potential risks and benefits of undertaking this medically complex and highly technical form of genetic testing.

There is much to learn about the process of patient education and informed consent for PGD and other new applications of genetic technology in reproductive medicine. Future research projects in this vein will include large-scale quantitative and qualitative research involving IVF/PGD providers, genetic counselors, and PGD patients to assess the content of informed consent materials and discussions, their experiences and opinions about the adequacy of informed consent processes that are in place for PGD, and how other clinical interactions beyond the informed consent process may inform patient decision making about IVF and PGD. In-depth analysis of these constituencies' understandings of the informed consent process would provide texture to the everyday practices of PGD and further guidance for ensuring adequate communication and informed consent for every patient.

With this study, our objective was to work toward closing the knowledge gap regarding clinical informed consent practices, and our conclusions are aimed toward remedying inconsistencies in clinical practice so to better serve the growing patient population seeking reproductive genetic testing via PGD. By securing adequate policies and practices for informed consent, patients will be better equipped to make health care decisions that are not only informed but also meet their needs, expectations, and beliefs about health and well-being while avoiding interventions that are undesirable or present an unacceptable degree of risk to them.

\section{ACKNOWLEDGMENTS}

This study was funded by a Seed Grant from The Center for Genetic Research Ethics and Law (NHGRI-ELSI Grant P50HG003390) at Case Western Reserve University.

\section{REFERENCES}

1. Gardner D, Weissman A, Howles C, Shoham Z. Textbook of assisted reproductive techniques: laboratory and clinical perspectives. London: Martin Duntz Ltd., 2001.

2. Braude P, Pickering S, Flinter F, Ogilvie CM. Preimplantation genetic diagnosis. Nat Rev Genet 2002;3:941-953.

3. Verlinsky Y, Cohen J, Munné S, et al. Over a decade of experience with preimplantation genetic diagnosis: a multicenter report. Fertil Steril 2004; 82:292-294.

4. Baruch S, Kaufman D, Hudson KL. Genetic testing of embryos: practices and perspectives of US in vitro fertilization clinics. Fertil Steril 2008;89: 1053-1058.

5. The Practice Committee of the American Society for Reproductive Medicine and the Practice Committee of the Society for Assisted Reproductive Technology. Elements to be considered in obtaining informed consent for ART. Fertil Steril 2006;86(suppl 4):S272-S2723.

6. Ensenauer R, Michels V, Reinke S. Genetic testing: practical, ethical, and counseling considerations. Mayo Clin Proc 1995;80:63-73.

7. Lewis CM, Pinel T, Whittaker JC, Handyside AH. Controlling misdiagnosis errors in preimplantation genetic diagnosis: a comprehensive model encompassing extrinsic and intrinsic sources of error. Hum Reprod 2001;16:43-50.

8. National Society of Genetic Counselors. Position Statement on Disclosure and Informed Consent (adopted 1991). Available at: http://www.nsgc.org/ about/position.cfm\#Disclosure. Accessed March 2, 2009

9. National Society of Genetic Counselors. Position Statement on Preconception/Prenatal Genetic Screening (adopted 2005). Available at: http://www. nsgc.org/about/position.cfm\#Preconception. Accessed March 2, 2009.

10. Thornhill AR, DeDie-Smulders CE, Geraedts JP, et al. ESHRE PGD Consortium 'Best practice guidelines for clinical preimplantation genetic diagnosis (PGD) and preimplantation genetic screening (PGS)'. Hum Reprod 2005;20:35-48.

11. Corveleyn A, Morris MA, Dequeker E, et al. Provision and quality assurance of preimplantation genetic diagnosis in Europe. Eur J Hum Genet 2008;16: 290-299.

12. Callum PA, Copperman A, Scott RT Jr, Levy B. Assessment of patient awareness of preimplantation genetic diagnosis (PGD) benefits and limitations. Fertil Steril 2005;84(suppl 1):S126-S127.

13. Kalfoglou A, Scott J, Hudson K. PGD patients' and providers' attitudes to the uses and regulation of preimplantation genetic diagnosis. Reprod Biomed Online 2005; 11:486-496.

14. Katz M, Fitzgerald A, Bankier A, Saulescu J, Cram DS. Issues and concerns of couples presenting for preimplantation genetic diagnosis (PGD). Prenat Diagn 2002;22:1117-1122

15. Lavery S, Aurell R, Turner C, et al. Preimplantation genetic diagnosis: patients' experiences and attitudes. Hum Reprod 2002;17:2464-2467.

16. Franklin S, Roberts C. Born and made: an ethnography of preimplantation genetic diagnosis. Princeton and Oxford: Princeton University Press, 2006.

17. Centers for Disease Control and Prevention. 2005 Assisted reproductive technology (ART) report: national summary. Atlanta: The Centers for Disease Control and Prevention, 2007. 